\title{
Expanding the Scope of JPE
}

\author{
Sean Shaheen \\ Editor-in-Chief, Journal of Photonics for Energy
}

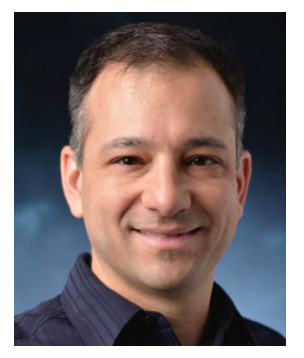

JPE is evolving to encompass new approaches and new ways of thinking about photonics for energy, which is reflected in the revised list of interest areas within our scope. ${ }^{1}$ It now includes energy conversion mechanisms that explicitly harness quantum phenomena. The desire to incorporate more quantum-focused content stems from the rapid advancement in quantum science and technology seen globally. Example topics include quantum coherence in vibronic or energy transfer mechanisms that may play a role in organic photovoltaic devices, ${ }^{2,3}$ photon up- and down-conversion, ${ }^{4}$ and quantum transduction. ${ }^{5}$ These and other topics are exciting for their ability to better approach fundamental limits of energy conversion based on quantum mechanical processes that can be extracted to macroscopic scale through clever engineering of materials, devices, and surrounding systems.

The scope also now includes the topics of artificial intelligence (AI) and neuromorphic computing. Even more rapidly than quantum science and technology, machine learning is growing in importance across basic science, engineering, and societal implementation. Stunning progress on Grand Challenge problems like protein folding ${ }^{6}$ is making even seasoned veterans of neural network research rethink the underlying concepts and potential applications of deep learning. JPE is interested in several facets of these areas as applied to energy science and technology. First, how can deep learning and other AI approaches be applied to better designing materials and devices for photonic energy conversion $?^{7,8}$ Second, how can photonic neuromorphic devices and optical systems be used for energy efficient computing? ?,10,11

We at JPE invite you to participate in the expansion of the journal's scope and to submit research articles in these new areas, in order to advance their fundamental understanding as well as to forge new directions for their technological applications in renewable energy. Quantum science and technology and neuromorphic engineering are rapidly evolving fields, and as such they often require better descriptions and specific goals and metrics that are applicable to the problem at hand. We invite you to apply your ingenuity and creativity in formulating these in your submissions to the journal.

\section{References}

1. “Scope," J. Photon. Energy, https://www.spiedigitallibrary.org/journals/journal-of-photonicsfor-energy/scope-and-details (accessed 30 March 2021).

2. Q. Bian et al., "Vibronic coherence contributes to photocurrent generation in organic semiconductor heterojunction diodes," Nat. Commun. 11, 617 (2020).

3. G. D. Scholes, "Quantum-coherent electronic energy transfer: did nature think of it first?" J. Phys. Chem. Lett. 1(1), 2-8 (2010).

4. D. H. Weingarten et al., "Experimental demonstration of photon upconversion via cooperative energy pooling," Nat. Commun. 8, 14808 (2017).

5. U.S. Department of Energy Office of Science, Basic Energy Sciences Roundtable, "Opportunities for basic research for next-generation quantum systems," 2017, https:// www.osti.gov/servlets/purl/1616258 (accessed 30 March 2021).

6. E. Callaway, “'It will change everything': DeepMind's AI makes gigantic leap in solving protein structures," Nature 588, 203-204 (2020).

7. N. Meftahi et al., "Machine learning property prediction for organic photovoltaic devices," npj Comput. Mater. 6, 166 (2020).

(C) 2021 Society of Photo-Optical Instrumentation Engineers (SPIE) 
8. ARPA-E, "Context-aware learning for inverse design in photovoltaics," 5 April 2019, https:// arpa-e.energy.gov/technologies/projects/context-aware-learning-inverse-design-photovoltaics (accessed 12 April 2021).

9. I. K. Schuller and R. Stevens, "Neuromorphic computing: from materials to systems architectures: report of a roundtable convened to consider neuromorphic computing basic research needs," Gaithersburg, MD, 2015, https://science.osti.gov/-/media/ascr/ pdf/programdocuments/docs/Neuromorphic-Computing-Report_FNLBLP.pdf (accessed 30 March 2021).

10. S. Chen, "Photonic chips for neuromorphic computing," SPIE News, 2020, https://spie.org/ news/photonic-chips-for-neuromorphic-computing (accessed 30 March 2021).

11. D. Dabos et al., "End-to-end deep learning with neuromorphic photonics," Proc. SPIE 11689, 1168901 (2021). 